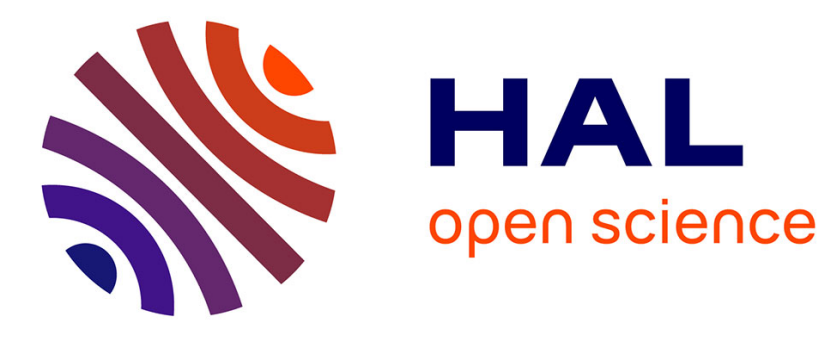

\title{
Underwater acoustic signals induced by intense ultrashort laser pulse
}

Yohann Brelet, Amélie Jarnac, Jérôme Carbonnel, Yves-Bernard André, André Mysyrowicz, Aurélien Houard, Dominique Fattaccioli, Régine Guillermin, Jean-Pierre Sessarego

\section{To cite this version:}

Yohann Brelet, Amélie Jarnac, Jérôme Carbonnel, Yves-Bernard André, André Mysyrowicz, et al.. Underwater acoustic signals induced by intense ultrashort laser pulse. Journal of the Acoustical Society of America, 2015, 137, pp.EL288. 10.1121/1.4914998 . hal-01138358

\section{HAL Id: hal-01138358 \\ https://hal-polytechnique.archives-ouvertes.fr/hal-01138358}

Submitted on 1 Apr 2015

HAL is a multi-disciplinary open access archive for the deposit and dissemination of scientific research documents, whether they are published or not. The documents may come from teaching and research institutions in France or abroad, or from public or private research centers.
L'archive ouverte pluridisciplinaire HAL, est destinée au dépôt et à la diffusion de documents scientifiques de niveau recherche, publiés ou non, émanant des établissements d'enseignement et de recherche français ou étrangers, des laboratoires publics ou privés. 


\title{
Underwater acoustic signals induced by intense ultrashort laser pulse
}

\author{
Yohann Brelet, Amélie Jarnac, Jérôme Carbonnel, Yves-Bernard André, \\ André Mysyrowicz, Aurélien Houard \\ Laboratoire d'Optique Appliquée, ENSTA ParisTech, Ecole Polytechnique, CNRS, 91762 Palaiseau, \\ France \\ aurelien.houard@ensta-paristech.fr,yohann.brelet@ipcms.unistra.fr,amelie.jarnac@fysik.lth.se, \\ jerome.carbonnel@ensta-paristech.fr, yves-bernard.andre@ensta-paristech.fr, \\ andre.mysyrowicz@ensta-paristech.fr \\ Dominique Fattaccioli \\ DGA/TN, Techniques navales, avenue de la Tour Royale, BP 40915, 83050 Toulon, France \\ dominique.fattaccioli@intradef.gouv.fr \\ and Régine Guillermin, Jean-Pierre Sessarego \\ Laboratoire de Mécanique et Acoustique, CNRS, 13402 Marseille, France \\ guillermin@lma.cnrs-mrs.fr, sessarego@lma.cnrs-mrs.fr
}

\begin{abstract}
Acoustic signals generated in water by terawatt (TW) laser pulses undergoing filamentation are studied. The acoustic signal has a very broad spectrum, spanning from 0.1 to $10 \mathrm{MHz}$, and is confined in the plane perpendicular to the laser direction. Such source appears to be promising for the development of remote laser based acoustic applications.

(C)2014 Acoustical Society of America
\end{abstract}

PACS numbers: 43.30.Lz, 43.25.Zx, 43.35.Ud, 43.30.Xm

Investigation of energy deposition in water from ultrashort laser pulses has attracted considerable interest in the last decade in view of potential applications ranging from laser surgery $^{1,2}$, to triggering of high voltage water switches ${ }^{3}$, or remote generation of acoustic sources $^{4-6}$. It has been recognized early on that an intense laser pulse focused in a transparent liquid can heat or ionize the medium, leading to the emission of an acoustic transient ${ }^{7,8}$. A laser-based method offers advantages in standoff underwater acoustics, in comparison with traditional sources consisting of arrays of hydro-acoustic transducers or sonars ${ }^{9,10}$. The first experiments were carried out with $\mathrm{CO}_{2}$, Neodymium glass and ruby laser sources, with pulse durations longer than $100 \mathrm{~ns}$. In this case, the laser induces a slow heating of the medium followed by a thermal expansion and emission of an acoustic wave ${ }^{11,12}$. Later, intense nanosecond pulses appeared to be more efficient, since they can easily generate optical breakdown in water through multiphoton and impact ionization ${ }^{13}$. After the rapid heating of the ionized volume, an explosive expansion occurs, yielding the emission of a shock wave ${ }^{14,15}$. Thus, fast lasers provide several orders of magnitude improvement in the conversion efficiency of the photon to acoustic energy if compared with the slow laser heating scheme ${ }^{16}$. With pulse durations in the sub-picosecond range, the ionization process being very fast, one expects the generation of a very broad acoustic wave.

In the present paper we experimentally report in situ space-time properties of the acoustical signal generated by a focused terawatt picosecond laser pulse in water. Several focusing 
geometries have been explored, under controlled laboratory conditions. The spectrum and directivity of the source were characterized, revealing a very broad emission ranging from 0.1 to $10 \mathrm{MHz}$ and a sharp directivity in elevation with respect to the laser beam axis. The properties of this acoustic source makes it particularly promising for sonar and imaging applications.

The laser used in the experiment is a mobile multi terawatt CPA (chirped pulse amplification) laser sytem (ENSTAmobile). It delivers transform-limited 50 fs pulses at 800 $\mathrm{nm}$ with energy of up to $290 \mathrm{~mJ}$ per pulse at a repetition rate of $10 \mathrm{~Hz}$. The laser beam (diameter FWHM $\sim 35 \mathrm{~mm}$ ) was focused in water by convex lenses with focal lengths of $f=$ 200 and $500 \mathrm{~mm}$, and crossed the air-water interface at normal incidence (see Fig. 1). The distance $h$ between the lens and the water surface was varied between 40 and $380 \mathrm{~mm}$. The laser pulse was temporally stretched up to $1 \mathrm{ps}$ by misalignment of the compressor stage of the chirped pulse amplifier to avoid damages on optical lenses, yielding a maximum peak power of $290 \mathrm{GW}$. Two hydrophones were used to monitor the underwater acoustic signal. A high frequency hydrophone (HF) Precision Acoustics $1.0 \mathrm{~mm}$ needle with a detection range $1 \mathrm{MHz}$ - $10 \mathrm{MHz}$ and a sensitivity of $-241.4 \mathrm{~dB}$ ref $1 \mathrm{~V} / \mu \mathrm{Pa}$ at $3 \mathrm{MHz}$. A low frequency hydrophone (LF) Reson TC4035 detecting between $50 \mathrm{kHz}$ and $800 \mathrm{kHz}$, with a sensitivity of $-215 \mathrm{~dB}$ ref $1 \mathrm{~V} / \mu \mathrm{Pa}$. A preamplification gain of $+20 \mathrm{~dB}$ was applied to the hydrophone signals. The LF and $\mathrm{HF}$ hydrophones were installed at distances $r$ larger than $80 \mathrm{~cm}$ and $30 \mathrm{~cm}$ from the laser axis respectively. Thus, both hydrophones were in the acoustic far field, determined as $r \geq$ $100 \lambda$ where $\lambda$ is the acoustic wavelength defined by $\lambda=v_{\text {water }} / F_{u s}$, where $F_{u s}$ is the ultrasound frequency and $v_{\text {water }}$ the speed of sound in water with $v_{\text {water }} \approx 1487 \mathrm{~m} / \mathrm{s}$ at $\mathrm{T}=21.6^{\circ} \mathrm{C}$. They could be moved longitudinally and collinearly with respect to the laser propagation axis using a motorized platform. The experimental setup is sketched in Fig. 1. The pool with dimension $13 \times 3 \times 2 \mathrm{~m}$ was filled with freshwater, at room temperature $\left(\mathrm{T} \sim 21.6^{\circ} \mathrm{C}\right)$.

Figures 2(a) and (b) present typical temporal waveforms measured by the hydrophones, when the laser pulse was focused in water with a lens $f=200 \mathrm{~mm}$ placed $40 \mathrm{~mm}$ above the water surface. The position $z$ of the hydrophones was adjusted to obtain the highest acoustic signal in the far field. The measured electric signals present an asymmetric $N$-shape ${ }^{17}$ revealing a steep positive pressure peaks followed by a negative pressure. The peak amplitude of this pressure wave in the far-field was found to decrease as $1 / r$, as shown in Fig. 3. Moreover, a signal-to-noise ratio (SNR) of $+40 \mathrm{~dB}$, for the HF hydrophone placed at $r \sim 31$ $\mathrm{cm}$ and $+25 \mathrm{~dB}$ for the LF hydrophone at $r \sim 80 \mathrm{~cm}$ have been deduced. The corresponding spectra for LF and HF hydrophones are plotted in Fig. 2(c). In both cases, the temporal signal has been deconvoluted from the hydrophone response and corrected for the preamplification gain. The spectrum of the acoustic signal is particularly broad, covering almost 10 octaves (precluding the band 0.8-1 MHz which is not covered). The equivalent pressure level of the signal at $1 \mathrm{~m}$ lies between $\sim 130$ and $\sim 160 \mathrm{~dB}$ ref $1 \mu \mathrm{Pa}$ at $1 \mathrm{~m}$ over the range $60 \mathrm{kHz}-6 \mathrm{MHz}$.

To get closer to undersea conditions, we injected small particles of kaolinite with a radius of a few tens of $\mu \mathrm{m}$ into water around the laser beam focal plane. The recorded signals exhibited a significant enhancement of the acoustic peak amplitude (up to $+20 \mathrm{~dB}$ ). Therefore 
one would expect that in a real undersea environment containing a large amount of impurities, the laser generated signals would also be stronger.

The dependence of the acoustic signal on elevation $\mathrm{z}$ has been studied with laser pulses of $290 \mathrm{~mJ}$ and duration of $1 \mathrm{ps}$ focused by a lens $f=200 \mathrm{~mm}$ at $h=40 \mathrm{~mm}$. A scan with $10 \mathrm{~mm}$ steps was performed along the $\mathrm{z}$ direction, in the plane containing the laser propagation axis, with $\mathrm{z}=0$ defined as the air-water interface. Taking into account the refraction of the beam at the air/water interface this height corresponds to geometrical focus positions $\mathrm{z} \approx 213 \mathrm{~mm}$. The shortest time-of-flight (ToF) is observed around $\mathrm{z}=220 \mathrm{~mm}$, close to the estimated geometrical focus of the laser beam. A similar behaviour is also observed with the LF hydrophone. Analogous results were obtained at different heights $h$, with a maximum acoustic signal always obtained when the detector position in $\mathrm{z}$ was close to the geometric focus of the laser pulse ${ }^{1}$.

The directivity of the optoacoustic source in the far-field was measured by recording signals in the (x,z) and (x,y) planes. Figure 4(a) displays the directivity diagrams in the vertical plane $(\mathrm{x}, \mathrm{z})$ for three ultrasonic frequencies. The diagrams present a main lobe enclosed within less than $2 \theta=10^{\circ}$, where $\theta$ is the angle formed between the positions $z$ of the acoustic source and that of the hydrophone along the $O x$ axis (see Fig. 1). The opening angle of the lobe experiences a small decrease as frequency increases. The observed slight disymetry with respect to the angle $0^{\circ}$ might by explained by an imperfect alignment with respect to the laser axis. We have determined that the acoustic source possesses a rotational symmetry in the plane $(\mathrm{x}, \mathrm{y})$. One then obtains the 3D emission diagram, as shown in Fig. 4(b), here for $\mathrm{F}_{\mathrm{us}}=2 \mathrm{MHz}$. The acoustic signal is null along the laser beam axis and maximal in its equatorial plane. This quasi 2-dimensional emission pattern is responsible for the $1 / r$ law of the pressure signal that we measured (see Fig. 3). To explain this feature, we note that the incident laser power exceeds by orders of magnitude the threshold power for filamentation. In this case, one expect the formation, close to the geometric focus, of superfilaments with elongated plasma column and densities exceeding by order of magnitude the plasma density found in a single filament ${ }^{18}$. Such a high density plasma column corresponds to a cylindric source responsible for the observed acoustic pattern in the far field. Note that shockwaves with a similar cylindrical shape were observed in ref. 15 in the vicinity of millimeter scale filaments produced in water.

In conclusion, we have demonstrated that focused ultrashort pulses with TW peak power can generate an optoacoustic source in water. Time of flight measurements reveal that it is localized close to the laser geometrical focus. Several characteristics of this optoacoustic source have also been derived, such as an interesting broad spectrum spanning at least from 0.1 to $10 \mathrm{MHz}$, with a pressure level of $160 \mathrm{~dB}$ ref $1 \mu \mathrm{Pa}$ at $1 \mathrm{~m}$ for $1 \mathrm{MHz}$ and a sharp directivity in the plane perpendicular to the laser propagation axis. This optoacoustic source may find applications in acoustic communications, or acoustic imaging.

\footnotetext{
${ }^{1}$ A secundary acoustic source generated at the air/water surface was also detected when the geometric focus of the lens gets closer to the interface (i.e. for $h$ large).
} 


\section{Acknowledgments}

This project has been supported by the French Direction Générale de l'Armement (grant $\mathrm{n}^{\circ}$ 066003600470750). The authors thank Laboratoire de Mécanique et Acoustique staff for technical assistance.

\section{References and links}

${ }^{1}$ K. Plamann, F. Aptel, C. L. Arnold, A. Courjaud, C. Crotti, F. Deloison, F. Druon, P. Georges, M. Hanna, J.-M. Legeais, F. Morin, E. Mottay, V. Nuzzo, D. A. Peyrot and M. Savoldelli, "Ultrashort pulse laser surgery of the cornea and the sclera," J. Opt. 12, 084002 (2010).

${ }^{2}$ A. Vogel, N. Linz, S. Freidank, and G. Paltauf, "Femtosecond-Laser-Induced Nanocavitation in Water: Implications for Optical Breakdown Threshold and Cell Surgery," Phys. Rev. Lett. 100, 038102 (2008).

${ }^{3}$ J. Woodworth, I. Molina, D. nelson, J. Maenchen, G. Sarkisov, J. Blickem, R. Starbird, F. Wilkins, D. Van DeValde and D. Johnson, "Green-laser-triggered Water Switching at 1.6 MV," IEEE Trans. Dielecr. Electr. Insul. 14, 951 (2007).

${ }^{4}$ T. G. Jones, A. Ting, J. Penano, P. Sprangle, and G. DiComo, "Remote Underwater Ultrashort Pulse Laser Acoustic Source," CThA1, CLEO/QELS 2006 (2006).

${ }^{5}$ W. Lauterborn and A. Vogel, in CF Delale (Ed.): Bubble Dynamics \& Shock Waves, SHOCKWAVES 8 (Springer-Verlag Berlin Heidelberg 2013). pp 67-103.

${ }^{6} \mathrm{~F}$. Blackmon and L. Antonelli, "Experimental demonstration of multiple pulse nonlinear optoacoustic signal generation and control," Appl. Opt. 44, 103 (2005).

${ }^{7}$ A. Vogel and W. Lauterborn, "Acoustic transient generation by laser-produced cavitation bubbles near solid boundaries," J. Acoust. Soc. Am. 84, 719 (1988).

${ }^{8}$ S. V. Egerev, "In search of a noncontact underwater acoustic source," Acoust. Phys. 49, 51-61 (2003).

${ }^{9}$ S. Sreeja, Ch. Leela, V. Rakesh Kumar, Suman Bagchi, T. Shuvan Prashant, P. Radhakrishnan, Surya P. Tewari, S. Venugopal Rao and P. Prem Kiran, "Dynamics of tightly focused femtosecond laser pulses in water,"

Laser Phys. 23, 106002 (2013).

${ }^{10}$ M. H. Helle, T. G. Jones, J. R. Penano, D. Kaganovich, and A. Ting, "Formation and propagation of meter-scale laser filaments in water," Appl. Phys. Lett. 103, 121101 (2013).

${ }^{11}$ N. Chotiros, "Nonlinear Optoacoustic Underwater Sound Source," Proc. SPIE 0925, Ocean Optics IX, 255 (1988).

${ }^{12}$ D. W. Tang, B. L. Zhou, H. Cao and G. H. He, "Dynamic thermal expansion under transient laser-pulse heating," Appl. Phys. Lett. 59, 3113 (1991).

${ }^{13}$ J. Noack and A. Vogel, "Laser-induced plasma formation in water at nanosecond to femtosecond time scales: calculation of thresholds, absorption coefficients, and energy density," IEEE Journ. Quant. Electro. 35, 1156 (1999).

${ }^{14}$ A. Vogel, J. Noack, K. Nahen, D. Theisen, S. Busch, U. Parlitz, D. X. Hammer, G. D. Noojin, B. A. Rockwell, and R. Birngruber, "Energy balance of optical breakdown in water at nanosecond to femtosecond time scales," Appl. Phys. B, 68, 271 (1999).

${ }^{15}$ F. V. Potemkin, E. I. Mareev, A. A. Podshivalov and V. M. Gordienko, "Laser control of filament-induced shock wave in water", Laser Physics Letters, 11, 106001 (2014).

${ }^{16}$ T. G. Jones, A. Ting, J. Penano, P. Sprangle, and L. D. Bibee, "Remote Intense Laser Acoustic Source," NRL Review, 121-123 ( 2007).

${ }^{17}$ R. T. Beyer, Nonlinear acoustics (Department of the Navy, Sea Systems Command, 1974).

${ }^{18}$ G. Point, Y. Brelet, A. Houard, V. Jukna, C. Milián, J. Carbonnel, Y. Liu, A. Couairon, and A. Mysyrowicz, "Superfilamentation in air", Phys. Rev. Lett. 112, 223902, 2014. 


\section{$\underline{\text { Captions and figures lists }}$}

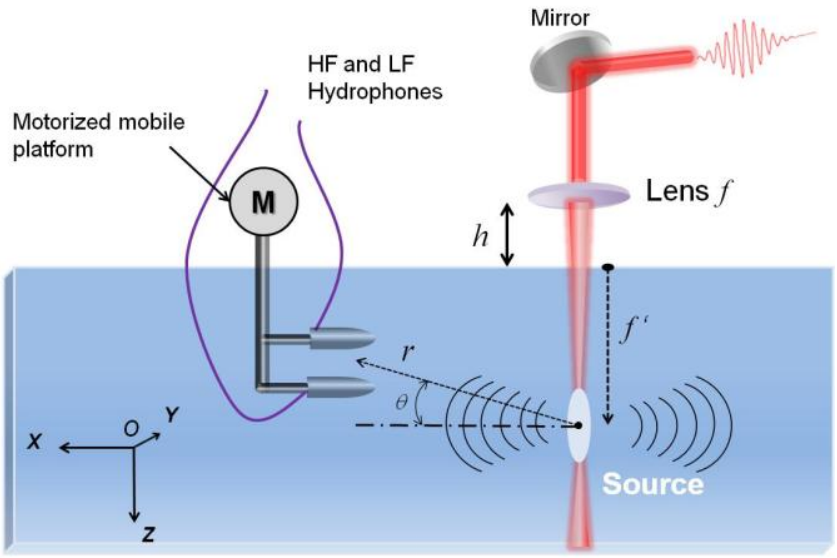

FIG. 1 (Color online). Experimental setup in the oceanic tank facility of Laboratoire de Mécanique et Acoustique.
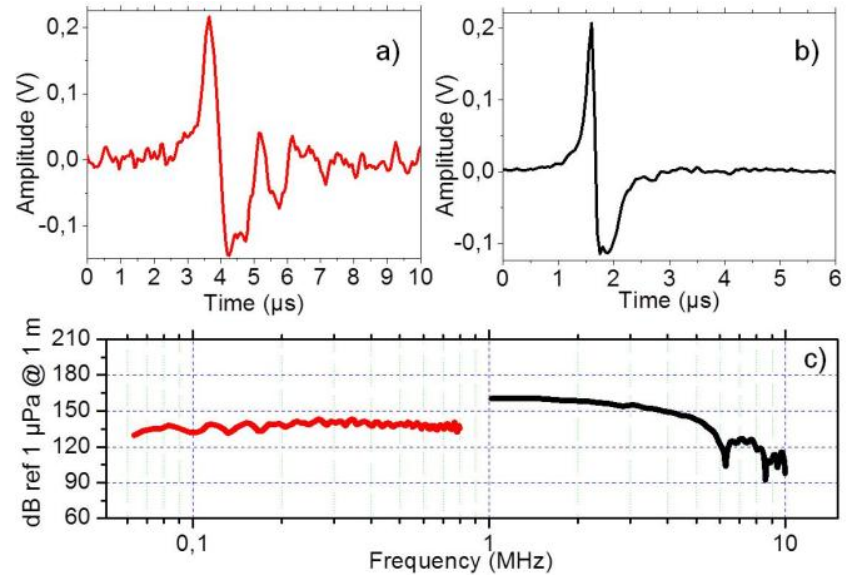

FIG. 2 (Color online). Electric signal detected by the LF (a) and HF (b) hydrophones at a depth $\mathrm{z} \approx 21 \mathrm{~cm}$ with a focusing lens $f=200 \mathrm{~mm}$ placed at a height $h=40 \mathrm{~mm}$. The sensors distance from the acoustic source was $r \approx 80$ and $31 \mathrm{~cm}$ for LF and HF, respectively. (c) Corresponding spectra with reference taken as $1 \mu \mathrm{Pa}$ at $1 \mathrm{~m}$ in water.

The origin of time corresponds to the inception of the transient acoustic source. 
Brelet et al:: JASA-EL

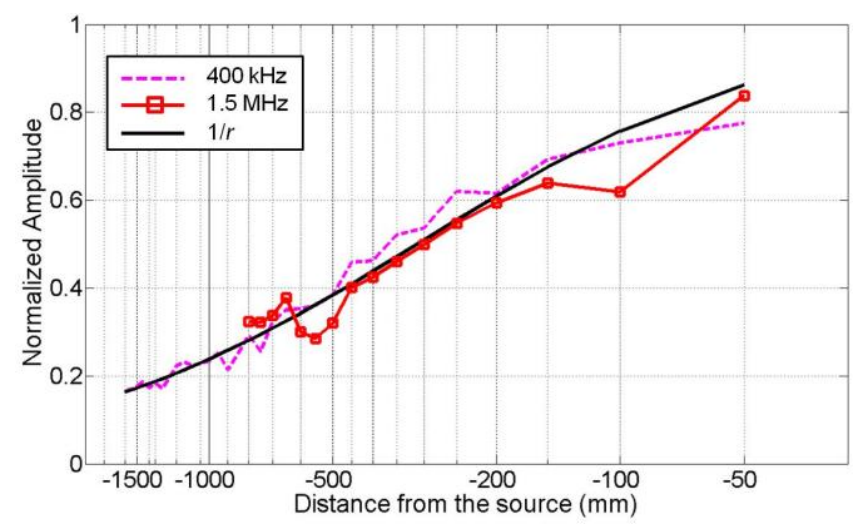

FIG. 3 (Color online). Pressure field dependance along $\mathrm{x}$-axis for two frequencies from LF and HF hydrophones, at a fixed depth $\mathrm{z} \approx 21 \mathrm{~cm}$ with a focusing lens $f=200 \mathrm{~mm}$ placed at a height $h=40 \mathrm{~mm}$. Black solid line shows $1 / r$ fit.
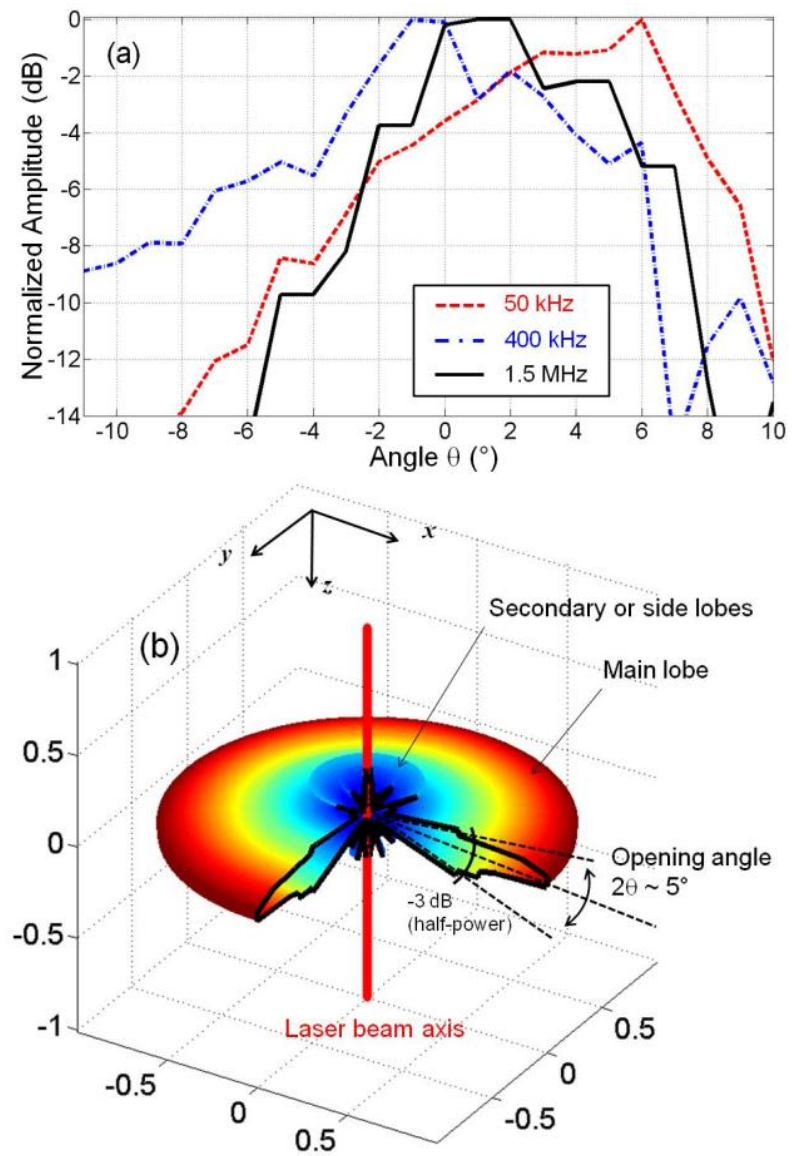

FIG. 4 (Color online). (a) Angular dependence of the acoustic signal at frequencies $F_{u s}=50 \mathrm{kHz}$ (dashed line), $400 \mathrm{kHz}$ (dash-dotted line) and $1.5 \mathrm{MHz}$ (solid line). (b) $3 \mathrm{D}$ diagram of the acoustic emission at $\mathrm{F}_{\mathrm{us}}=2 \mathrm{MHz}$. Laser parameters are $\mathrm{E}_{\mathrm{inc}}=290 \mathrm{~mJ}, \mathrm{t}_{\mathrm{p}} \sim 1 \mathrm{ps}, f=+200 \mathrm{~mm}$ and $h \sim 40 \mathrm{~mm}$. 